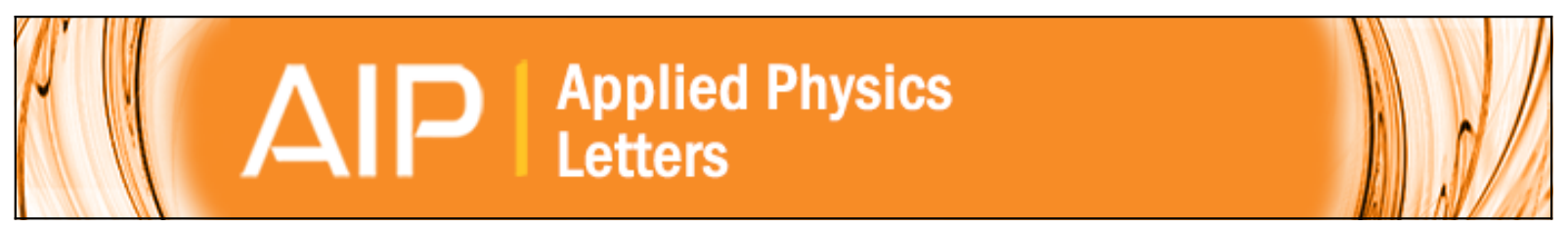

Micromagnetic modeling of experimental hysteresis loops for heterogeneous electrodeposited cobalt films

Matthew P. Seymour, lan Wilding, Ben Xu, Jason I. Mercer, Martin L. Plumer, Kristin M. Poduska, Anand Yethiraj , and Johan van Lierop

Citation: Applied Physics Letters 102, 072403 (2013); doi: 10.1063/1.4793209

View online: http://dx.doi.org/10.1063/1.4793209

View Table of Contents: http://scitation.aip.org/content/aip/journal/apl/102/7?ver=pdfcov

Published by the AIP Publishing

AlP 


\title{
Micromagnetic modeling of experimental hysteresis loops for heterogeneous electrodeposited cobalt films
}

\author{
Matthew P. Seymour, ${ }^{1}$ Ian Wilding, ${ }^{1}$ Ben Xu, ${ }^{1}$ Jason I. Mercer, ${ }^{1}$ Martin L. Plumer, ${ }^{1}$ \\ Kristin M. Poduska, ${ }^{1}$ Anand Yethiraj, ${ }^{1}$ and Johan van Lierop ${ }^{2}$ \\ ${ }^{1}$ Department of Physics and Physical Oceanography, Memorial University of Newfoundland, \\ St. John's, Newfoundland AlB 3X7, Canada \\ ${ }^{2}$ Department of Physics and Astronomy, University of Manitoba, Winnipeg, Manitoba R3T 2N2, Canada
}

(Received 12 September 2012; accepted 6 February 2013; published online 20 February 2013)

\begin{abstract}
Micromagnetic modeling provides a realistic description of the magnetic switching behavior in electrodeposited Co thin films that are either uniform (untemplated) or templated with an array of sub-micron spheres. Quantitative agreement between experimental results and simulations based on the Landau-Lifshitz-Gilbert equations is achieved for both in-plane and perpendicular $M H$ loops at two temperatures. By accounting for the sweep-rate dependence in coercivity values from simulated loops (with sweep rates $10^{4}-10^{-1} \mathrm{Oe} / \mathrm{ns}$ ) and then extrapolating to the experimental regime (measurement times of $10-100 \mathrm{~s}$ ), a self-consistent set of microscopic parameters is established to accommodate the complexity of the electrodeposited films. (c) 2013 American Institute of Physics. [http://dx.doi.org/10.1063/1.4793209]
\end{abstract}

Micromagnetic modeling based on the Landau-LifshitzGilbert (LLG) equations of magnetization $(M)$ vs. applied magnetic field $(H)$ loops is a popular and useful means to characterize and understand the field-induced switching properties of thin films and other nanoscale systems. Simulation results are often matched with experimental data in an attempt to assess microscopic materials properties such as magnetocrystalline anisotropy, exchange interactions, and grain size. The distributions of these properties are especially important for assessing recording media. In order to increase confidence that modeled properties are useful and correct, one needs to consider and account for an under-appreciated aspect of the modeling: the intrinsic differences in the magnetic switching processes between an in-plane and a perpendicular applied magnetic field. ${ }^{1}$ For a rigorous test, we examined electrodeposited films, both uniform and microscopically templated, which are known to have a large distribution of magnetocrystalline anisotropy values and grain sizes. ${ }^{2}$

A fundamental limitation in comparing simulated and experimental $M H$ loops arises from time-scale differences in acquiring the data, as reflected by the sweep rate, $R$. Whereas an experimental $M H$ loop is constrained to relatively low sweep rates $\left(R \sim 10^{-8} \mathrm{Oe} / \mathrm{ns}\right)$ due to unavoidable effects of solenoid inductance and finite magnetic field stabilization times, LLG modeling with ps time steps limits sweep rates to be many orders of magnitude faster $\left(R \sim 10^{3} \mathrm{Oe} / \mathrm{ns}\right)$ to achieve reasonable computation times. Although $R$-dependent effects are unimportant at low temperatures (for which thermal and field energies are significantly less than the magnetocrystalline anisotropy energy), their impact can be dramatic at higher temperatures. Most notably, coercivity $\left(H_{c}\right)$ is widely believed to follow Arrenhius-Néel behavior (for noninteracting particles) as manifest in Sharrock's law. ${ }^{3}$ This means that $H_{c}$ values decrease as $R$ gets smaller (especially at high temperatures), and this is a well known issue when comparing model results with experiment. ${ }^{4,5}$ Indeed, to provide quantitative comparison between model and experiment, the non-trivial accounting of the $H_{c}-R$ dependence and simultaneous simulation of in-plane and perpendicular $M H$ loops to obtain valid microscopic parameters and meaningful agreement at and above room temperature are required. ${ }^{6}$

Useful application of LLG models is especially significant for bit-patterned magnetic media, where $M H$ loops have been used to evaluate schemes for fabrication, including dot or antidot arrays. ${ }^{7-9}$ We consider here matching simulations with experiment by working on a challenging system of uniform and patterned antidot thin films fabricated by electrodepositing cobalt through spin-coated colloidal templates. While lithographic methods tend to be time consuming and resource intensive, ${ }^{10,11}$ the production of evaporatively selfassembled colloidal templates is a simpler and lower-cost alternative. For example, Zhukov et al ${ }^{12,13}$ electroplated a number of magnetic materials through evaporatively formed latex sphere arrays. They examined the dependence of $H_{c}$ on film thickness relative to template (sphere) diameter, emphasizing the interplay between magnetic domain wall widths and void dimensions. The comparison of in-plane experimental and micromagnetic modeled loops of antidot arrays has been attempted by others, but with mixed results. ${ }^{14,15}$

Despite the simplicity of the evaporative production of sphere arrays, continuous and crack-free templates have proven difficult to achieve over the macroscopic length scales that are relevant for hard disks. In this context, we and others have investigated the viability of crack-free polycrystalline colloidal templates prepared by a very rapid spin coating method. ${ }^{16-19}$ For our work, Co was electrodeposited on indium tin oxide (ITO) coated glass slides (Delta Technologies) using an electrolyte containing $0.1 \mathrm{M} \mathrm{H}_{3} \mathrm{BO}_{3}$ (EM Science, $99.5 \%$ pure) and $0.1 \mathrm{M} \mathrm{CoSO}_{4}$ (EM Science, 97.0\% pure) with application of a constant potential (Hokuto Denko HA 501 potentiostat/galvanostat, $-1.1 \mathrm{~V}$ to $-1.25 \mathrm{~V}$ vs. saturated calomel) for times up to $10 \mathrm{~min}$ to yield deposits with average thicknesses $\sim 100 \mathrm{~nm}$. To produce patterned films, we fabricated porous silica templates by spin-coating silica spheres, and then we electroplated cobalt in the voids between the particles. Template thickness was controlled via 
the substrate rotation rate, as described elsewhere. ${ }^{18}$ In order to fabricate the porous templates, we utilized silica spheres $(250 \mathrm{~nm}$ or $500 \mathrm{~nm}$ diameter, Fiber Optic Center Inc.) that were dried for at least $3 \mathrm{~h}$ at $150^{\circ} \mathrm{C}$ to remove absorbed water. Spheres were then mixed with methyl ethyl ketone (MEK) at a ratio of $7.2 \mathrm{ml}$ of MEK for each gram of silica (equivalent to a mass fraction of 5.8 MEK to silica). The suspension was ultrasonicated for several hours in order to disperse the spheres. For evaporative spincoating, $37 \mu \mathrm{l}$ of the colloidal suspension was dropped, using a pipettor, onto ITO coated cover slides $(25 \mathrm{~mm} \times 25 \mathrm{~mm})$, at the center of rotation and after the substrate reached a speed of 3000 RPM. Within seconds, the MEK evaporated to leave a single layer of spheres behind.

Atomic force and magnetic force microscopy (AFM/ MFM, Asylum Research MFP-3D) data (Fig. 1) showed that the electrodeposit was composed of distinct grains with considerable height variation. Typically, the grains were smaller than the sphere diameter. As a result, it was necessary to model the cobalt electrodeposit as a film composed of discrete grains (nanocrystallites) rather than as a continuous film. Measurements of $M H$ loops were made at 10, 100, and $300 \mathrm{~K}$ using either a Quantum Designs Magnetic Property Measurement System SQUID magnetometer or a Microsense EV-9 Vibrating Sample Magnetometer. Typical sample dimensions were $4 \mathrm{~mm} \times 8 \mathrm{~mm}$. All magnetic data were corrected for contributions from the ITO substrate. Although the films were prepared in an aqueous environment under ambient atmospheric conditions, no evidence of cobalt oxide was observed; there was no exchange bias visible in 300 to $10 \mathrm{~K} 50 \mathrm{kOe}$ field-cooled $M H$ loops. ${ }^{20}$

Zero-temperature simulations were performed using commercial LLG software. ${ }^{21}$ Uniform films were modelled as $10 \times 10 \mathrm{~nm}^{2}$ laterally dimensioned cells, with $30 \mathrm{~nm}$ thickness, on a square lattice using $16 \times 16$ cells with periodic boundary conditions imposed in the plane. For the patterned

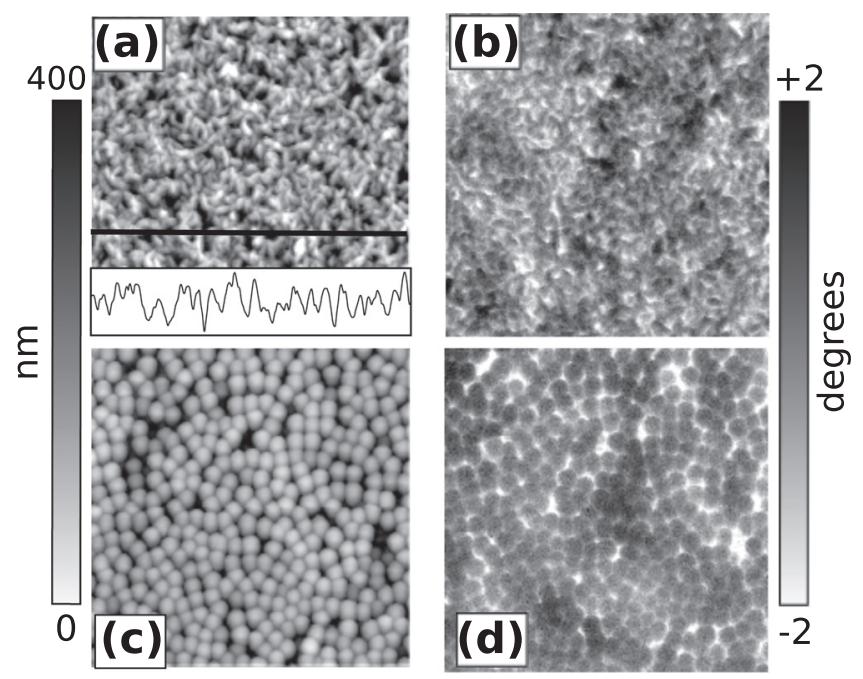

FIG. 1. Representative AFM (left panels) and MFM (right panels) images for untemplated [(a) and (b)] and templated [(c) and (d)] cobalt electrodeposits. The height scale for the AFM images [(a) and (c)] covers $400 \mathrm{~nm}$, with white corresponding to the tallest features. The MFM images [(b) and (d)] cover a phase range of $4^{\circ}$. Each image covers an area of $10 \mu \mathrm{m} \times 10 \mu \mathrm{m}$. Panel (a) also includes a height cross section, taken at the position of the black horizontal line, with a total height scale of $250 \mathrm{~nm}$. film, $250 \mathrm{~nm}$ adjacent cylinders of non-magnetic material were arranged in a square array, with Co in between, using $50 \times 50$ cells. Thicker films $(60 \mathrm{~nm})$ required more timeconsuming simulations, and showed no appreciable changes in the calculated $M H$ loops. Simulations assumed a typical Co saturation magnetization $\left(M_{s}=1400 \mathrm{emu} / \mathrm{cm}^{3}\right)$. The best agreement with the experimental data was obtained with a uniaxial magnetocrystalline anisotropy $K=2.0 \times 10^{6}$ $\mathrm{erg} / \mathrm{cm}^{3}, \sim 2.5 \times$ smaller than typical bulk values. ${ }^{22}$ Since the AFM cross-section in Fig. 1 indicates that the Co nanocrystallites are well separated, simulations assumed that there was no exchange interaction between crystallites. Rather large Gaussian distributions (with 20\% standard deviations) gave the best match to the experimental data. The directions of the anisotropy axes were taken to be uniformly random, as done in the previous studies of nanoparticles. ${ }^{23}$ Zero temperature simulations used a damping factor of $\alpha=0.2$, time step of $0.5 \mathrm{ps}$, and the convergence factor on the cell moments was $10^{-4}$.

Figure 2(a) shows good agreement for uniform films between the experimental data taken at $100 \mathrm{~K}$ and simulated results at $0 \mathrm{~K}$ for both in-plane and perpendicular $M H$ loops. Thermally activated sweep-rate dependence is not expected to
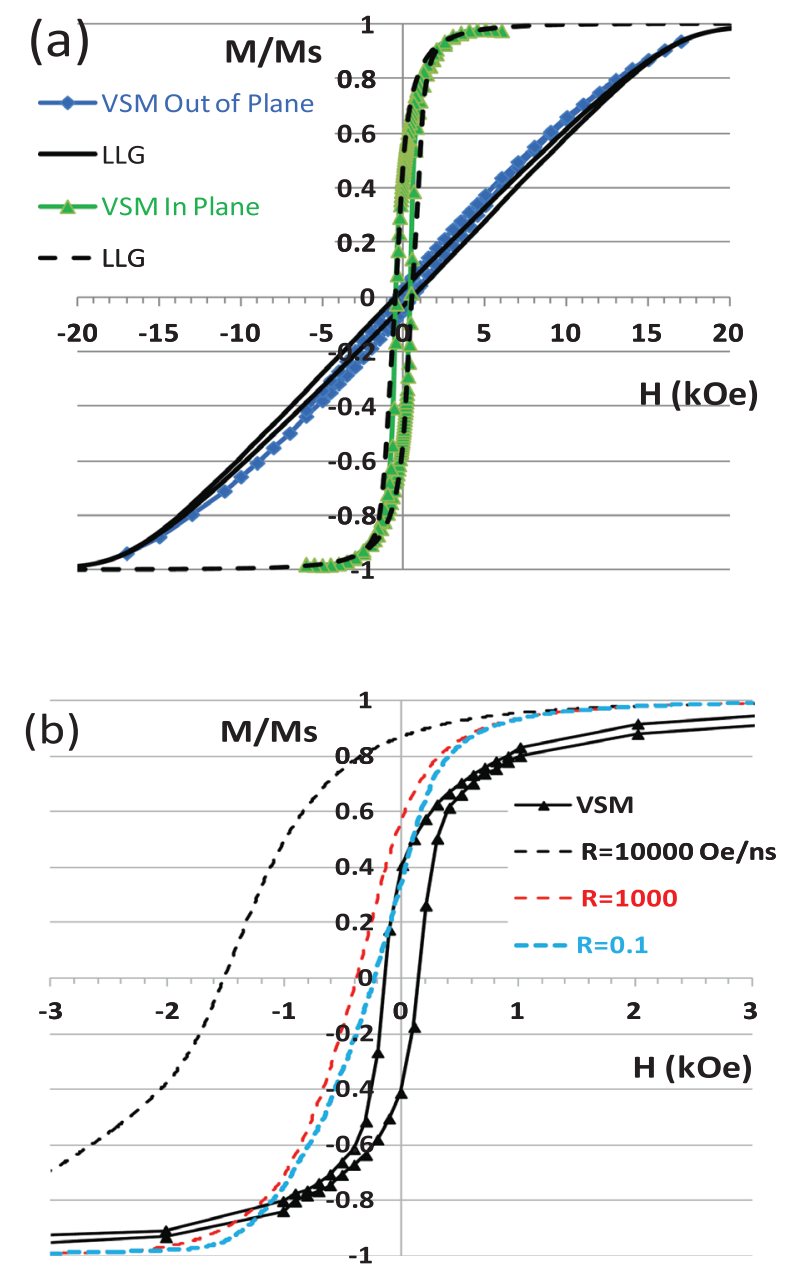

FIG. 2. (a) Uniform cobalt films: Comparison of experimental and simulated in-plane and perpendicular $M H$ loops at low temperatures. (b) In-plane uniform film experimental and simulated (half-loop) results at $300 \mathrm{~K}$ for different $R$. The simulation with lowest $R$ most closely matched the experimental results (black triangles). 
be a factor at these temperatures for high-anisotropy cobalt. Experimental coercivity values are similar for both field orientations $\left(H_{c} \sim 500 \mathrm{Oe}\right)$ and are well-matched by the simulation results. This confirms the premise that the cobalt magnetic anisotropy axes have no preferred directions. In Figure 2(a), the perpendicular hysteresis loop (blue diamonds) exhibits both a shallower slope and a larger saturation field than the in-plane case (green triangles). This is due to the thin film's magnetostatic shape anisotropy, which is well-captured by the simulations (solid and dashed lines for in-plane and perpendicular cases, respectively). The rounding of the curves is also a signature of the effect of distributions in saturation moment, and in anisotropy direction and magnitude.

At room temperature, there are significant discrepancies between experiment and simulations, and these differences can be explained in terms of sweep rate $R$ effects. Demonstrating the effect with uniform films, Fig. 2(b) compares the results of simulations at $300 \mathrm{~K}$ (both in-plane and perpendicular) with experimental $M H$ loops. A reduced time step of 0.2 ps and $\alpha=0.1$ were used, along with a reduced anisotropy $K=1.0 \times 10^{6} \mathrm{erg} / \mathrm{cm}^{3}$ to approximate intrinsic temperature effects of a thin film between 0 and $300 \mathrm{~K}^{22}$ The field was changed in steps of $\Delta H=100 \mathrm{Oe}$ and the simulation run for a time of $\Delta t$ at each field value, defining the sweep rate $R=\Delta H / \Delta t$. Reducing the time step further to $0.1 \mathrm{ps}$ had no effect on the results. Six different sweep rates from $R=10^{4} \mathrm{Oe} / \mathrm{ns}$ to $R=0.1 \mathrm{Oe} / \mathrm{ns}$ were performed, and the longer runs (at $R=0.1 \mathrm{Oe} / \mathrm{ns}$ ) took about 6 days on a typical workstation (with each slower decade of $R$ taking ten times longer to run). For these simulations, a new efficient UNIX-based LLG algorithm was employed and run on multiple central processing units. ${ }^{24}$ Standard LLG simulations at experimental time scales $\left(R \sim 10^{-8} \mathrm{Oe} / \mathrm{ns}\right)$ have impractically long run times; however, a semi-analytic model for this purpose has recently been developed which combines an energy barrier calculation along with thermal Monte Carlo simulations. ${ }^{25}$ Fig. 2(b) shows representative results and the corresponding experimental data for an in-plane applied field. The simulated loops trend toward the experimental results more closely as $R$ decreases.

A summary of $H_{c}$ vs. $R$ extracted from simulations are presented in Fig. 3, with error bars estimated from the scatter in multiple simulations $(15-100)$ at the same value of $R$. For slower sweep-rates, there is nearly logarithmic scaling of $H_{c}$ with $R$, consistent with Arrhenius-type thermally activated switching. Simulations on related magnetic thin-film systems suggest that this scaling holds, even at long time scales, in cases of weak inter-particle interactions. ${ }^{6,25}$ Extrapolation to experimental times scales $\left(R=10^{-8} \mathrm{Oe} / \mathrm{ns}\right)$ has a large associated error, yielding $H_{c}=190 \pm 100$ Oe for the in-plane loops and $H_{c}=120 \pm 100$ Oe for the out-of-plane loops. Nevertheless, these values are consistent with the experimental result that $H_{c}=150 \pm 10$ Oe for both field orientations for uniform films at $300 \mathrm{~K}$.

As a further test, we investigated the more complicated case of the templated electrodeposits. Even though the templated electrodeposits are far from perfect arrays, the patterned films were simulated surprisingly well using a highly idealized model based on a square array of touching, cylindrical non-magnetic regions. This simplistic model assumes that

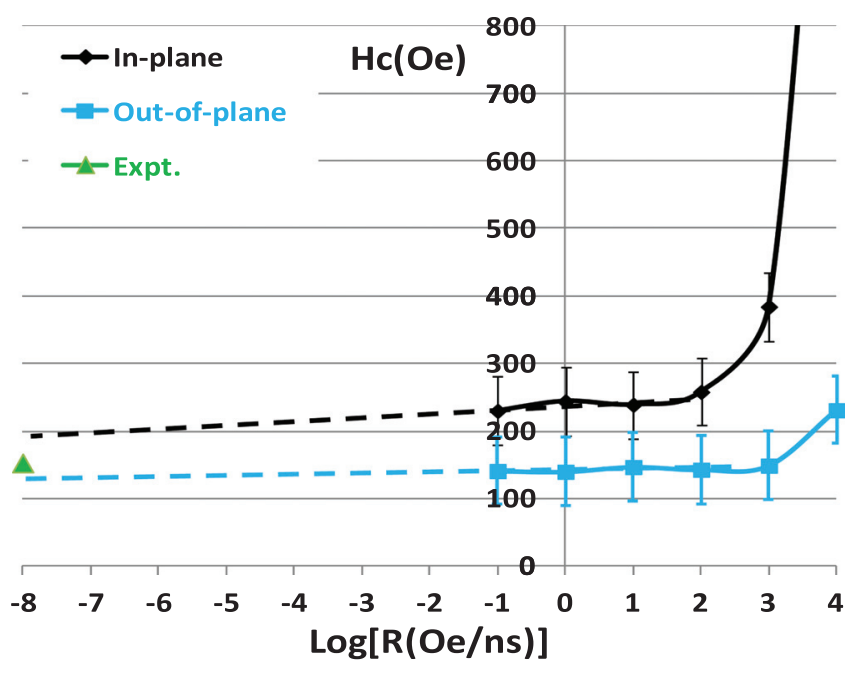

FIG. 3. Simulated results for coercivity vs. $\log$ sweep rate for uniform films at $300 \mathrm{~K}$. In-plane coercivity at $\log (\mathrm{R})=4 \mathrm{Oe} / \mathrm{ns}$ has the average value of 1500 Oe (off scale).

the principal effect of the non-magnetic regions is to modify the magnetostatic interaction between grains by introducing circular shape anisotropy near the voids. Fig. 4(a) compares in-plane and perpendicular loops simulated with $250 \mathrm{~nm}$
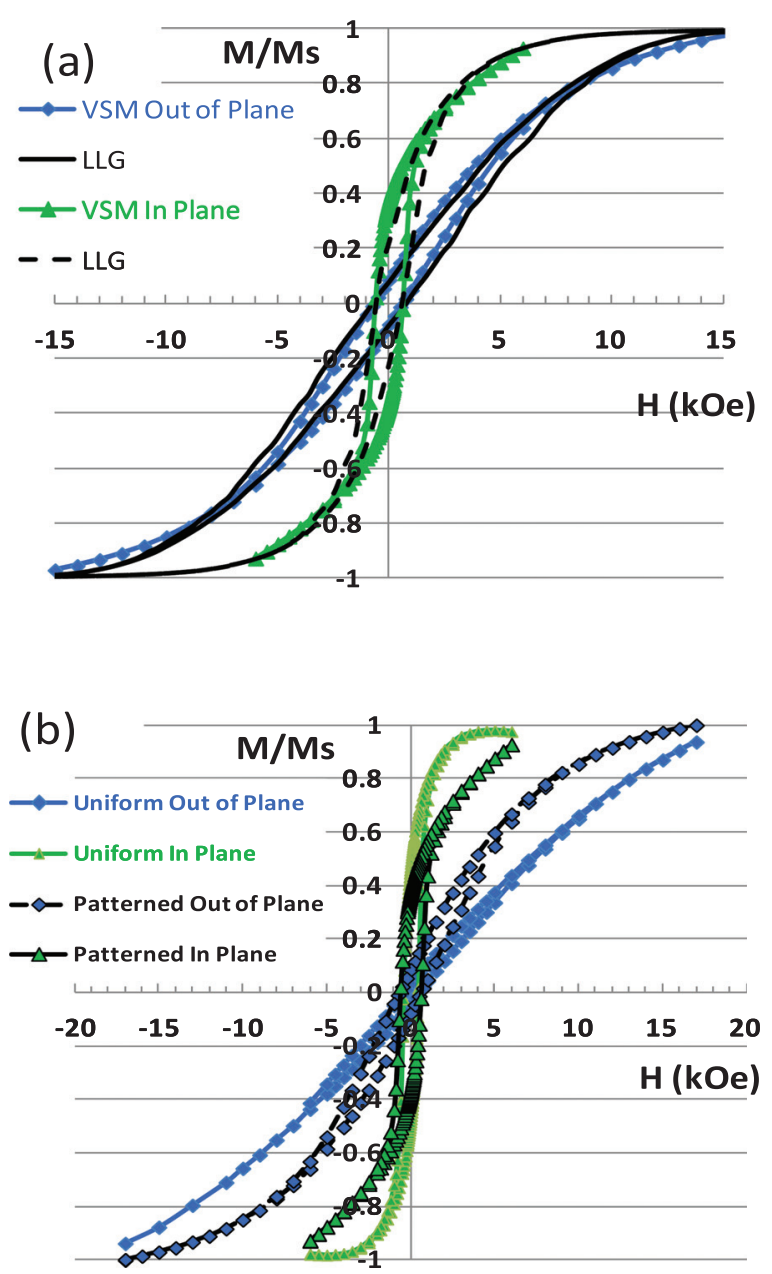

FIG. 4. (a) Patterned cobalt films: Simulated results for patterned films at zero temperature and experimental loops at $100 \mathrm{~K}$. (b) Comparison of experimental uniform and patterned cobalt films at $100 \mathrm{~K}$. Perpendicular loops were normalized to the maximum $\mathrm{M}$ value, obtained at $18 \mathrm{kOe}$. 
diameter cylinders at $\mathrm{T}=0 \mathrm{~K}$ with experimental data from a $250 \mathrm{~nm}$ sphere patterned anti-dot film at $100 \mathrm{~K}$. At this lower temperature, the effects of sweep rate are negligible since the probability for grain switching $\sim e^{-K V / k_{B} T}$ is very much smaller at $100 \mathrm{~K}$ compared to $300 \mathrm{~K}$. Clearly, magnetic domain reversal from thermal energy during a field increment is statistically insignificant. The results for the two applied field directions are more similar in patterned films than in the uniform films (Fig. 4(b)). The removal of magnetic material due to templating reduces magnetostatic effects, which leads to different domain structures near the anti-dots. There is also an enhancement of $H_{c} \sim 600 \mathrm{Oe}$. Similar effects have been observed in other magnetic systems. ${ }^{23}$

In summary, the goal of this work was to demonstrate that relevant microscopic parameters involved in LLG simulations of thin magnetic films can be determined reliably if care is taken to compare with a number of experimental results. The challenge of realizing an accurate micromagnetic model of $\mathrm{MH}$ loops in the case of complicated electroplated cobalt films has been realized. The remarkably good agreement between the simulations and data for both uniform and anti-dot patterned films at low temperatures is seen for the field applied both in plane and out of plane, has been achieved mainly by taking into account distributions in fundamental parameters. Room-temperature sweep-rate dependence giving rise to a reduction in the coercivity at room temperature was also simulated. This approach could easily be generalized to other systems so that future experimental studies could avail of LLG modelling to help understand and perhaps predict magnetic hysteresis responses.

We acknowledge the Natural Science and Engineering Resource Council of Canada (NSERC), the Canada Foundation for Innovation (CFI), and the Atlantic Computational Excellence Network (ACEnet) for funding.
We also thank J. P. Whitehead for assistance with the interpretation of simulation results.

${ }^{1}$ M. L. Plumer, M. C. Rogers, and E. Meloche, IEEE Trans. Magn. 45, 3942 (2009).

${ }^{2}$ T. Chen and P. Cavallotti, Appl. Phys. Lett. 41, 205 (1982).

${ }^{3}$ X. Feng and P. B. Visscher, J. Appl. Phys. 95, 7043 (2004).

${ }^{4}$ J. Xue and R. H. Victora, Appl. Phys. Lett. 77, 3432 (2000).

${ }^{5}$ X. H. Tan, S. J. Collocott, and H. Xu, J. Magn. Magn. Mater. 324, 2565 (2012).

${ }^{6}$ M. L. Plumer, M. D. Leblanc, J. P. Whitehead, and J. van Ek, J. Appl. Phys. 111, 123905 (2012).

${ }^{7}$ C. Choi, K. Noh, Y. Oh, C. Kuru, D. Hong, L.-H. Chen, S.-H. Liou, T.-Y. Seong, and S. Jin, IEEE Trans. Magn. 47, 2532 (2011).

${ }^{8}$ K. Zhang and D. Wei, J. Magn. Magn. Mater. 324, 276 (2012).

${ }^{9}$ B. D. Terris, J. Magn. Magn. Mater. 321, 512 (2009).

${ }^{10}$ S. Nakagawa, S. Takayama, and M. Naoe, IEEE Trans. Magn. 33, 3091 (1997).

${ }^{11}$ M. Plumer, J. van Ek, and D. Weller, The Physics of Ultra-High-Density Magnetic Recording (Springer-Verlag, 2001).

${ }^{12}$ A. A. Zhukov, A. V. Goncharov, P. A. de Groot, P. N. Bartlett, and A. G. Mohamed, J. Appl. Phys. 93, 7322 (2003).

${ }^{13}$ A. A. Zhukov, A. V. Goncharov, P. A. de Groot, A. G. Mohamed, P. N. Bartlett, R. Boardman, H. Fangohr, V. Novosad, and G. Karapetrov, Appl. Phys. Lett. 88, 062511 (2006).

${ }^{14}$ V. P. Chuang, W. Jung, C. A. Ross, J. Y. Cheng, O.-H. Park, and H.-C. Kim, J. Appl. Phys. 103, 074307 (2008).

${ }^{15}$ C.-C. Ho, T.-W. Hsieh, H.-H. Kung, W.-T. Juan, K.-H. Lin, and W.-L. Lee, Appl. Phys. Lett. 96, 122504 (2010).

${ }^{16}$ P. Jiang and M. J. McFarland, J. Am. Chem. Soc. 126, 13778 (2004).

${ }^{17}$ A. Mihi, M. Ocaña, and H. Míguez, Adv. Mater. 18, 2244-2249 (2006).

${ }^{18}$ C. Arcos, K. Kumar, W. González-Viñas, R. Sirera, K. M. Poduska, and A. Yethiraj, Phys. Rev. E 77, 050402 (2008).

${ }^{19}$ M. Giuliani, W. González-Viñas, K. M. Poduska, and A. Yethiraj, J. Phys. Chem. Lett. 1, 1481 (2010).

${ }^{20}$ W. Meiklejohn and C. Bean, Phys. Rev. 105, 904 (1957).

21 "LLG Micromagnetics Simulator" (unpublished).

${ }^{22}$ B. D. Cullity, Introduction to Magnetic Materials (Addison-Wesley, 1972).

${ }^{23}$ M. L. Plumer, J. van Lierop, B. W. Southern, and J. P. Whitehead, J. Phys.: Condens. Matter 22, 296007 (2010).

${ }^{24}$ J. I. Mercer and J. P. Whitehead, "MagLua: A general purpose, parallel, scriptable micromagnetics simulator with graphics processing unit acceleration," J. Comp. Phys. (in preparation).

${ }^{25}$ T. J. Fal, J. I. Mercer, M. Le Blanc, J. P. Whitehead, M. L. Plumer, and J. van Ek, Phys. Rev. B 87, 064405 (2013). 